\title{
A Retrospective Analysis of the Effects of TIME on Compliance and Driving Pressures in Acute Respiratory Distress Syndrome: The TIMED Study
}

Nikhil Jagan ( $\nabla$ nikhiljagan@creighton.edu )

$\mathrm{CHI}$ Health Creighton University Medical Center - Bergan Mercy: CHI Health Creighton University Medical Center Bergan Mercy https://orcid.org/0000-0001-8494-1622

\section{Lee Morrow}

Creighton University

Ryan Walters

Creighton University

Robert Plambeck

Creighton University

Ian $\mathbf{N g}$

Creighton University

Jasmin Chovatiya

Creighton University

Jeffrey Macaraeg

Creighton University

Karson Kalian

Creighton University

Zachariah Wittenberg

Creighton University

William Pruett

Creighton University

Jonathan Knedler

$\mathrm{CHI}$ Health

\section{Lauren Klein}

$\mathrm{CHI}$ Health

\section{Pooja Kasinath}

Creighton University

\section{Emily Dyer}

Creighton University

\section{Adam Bergh}

Creighton University 
Mark Malesker

Creighton University

\section{Research}

Keywords: Acute respiratory distress syndrome, Static compliance, Driving pressures, Noninvasive ventilation

Posted Date: November 18th, 2021

DOI: https://doi.org/10.21203/rs.3.rs-1040418/v1

License: (c) (i) This work is licensed under a Creative Commons Attribution 4.0 International License. Read Full License 


\section{Abstract}

\section{Background:}

The evolution of compliance and driving pressure in acute respiratory distress syndrome (ARDS) and the effects of time spent on noninvasive respiratory support prior to intubation has not been well studied. We conducted this study to assess the effect of the duration of noninvasive respiratory support prior to intubation (i.e., Noninvasive ventilation (NIV), High flow nasal cannula (HFNC), or a combination of NIV and HFNC) on static compliance and driving pressure and retrospectively describe its trajectory over time for COVID-19 and non-COVID-19 ARDS while on mechanical ventilation.

\section{Methods:}

Retrospective analysis of prospectively collected data from one university-affiliated academic medical center, one a rural magnet hospital, and three suburban community facilities. A total of 589 patients were included: 55 COVID-19 positive, 137 culture positive, and 397 culture negative patients. Static compliance and driving pressure were calculated at each 8-hour ventilator check.

\section{Results:}

Days of pre-intubation noninvasive respiratory support was associated with worse compliance and driving pressure but did not moderate any trajectory. COVID-19 positive patients showed non-statistically significant worsening compliance by 0.08 -units per ventilator check $(p=.241)$, whereas COVID-19 negative patients who were either culture positive or negative patients showed statistically significant improvement ( 0.12 and 0.18 , respectively; both $p<.05)$; a statistically similar but inverse pattern was observed for driving pressure.

\section{Conclusion}

In contrast to non-COVID-19 ARDS, COVID-19 ARDS was associated with a more ominous trajectory with no improvement in static lung compliance or driving pressures. Though there was no association between days of pre-intubation noninvasive respiratory support and mortality, its use was associated with worse overall compliance and driving pressure.

\section{Introduction}

ARDS has been a clinical challenge since its original description in $1967^{1}$. Current management of ARDS patients involve supportive mechanical ventilation strategies to correct the underlying hypoxemia ${ }^{2,3}$. Reduced compliance is a hallmark of ARDS, largely reflecting the degree of volume loss of the lung but is not part of the current definition of ARDS as it was not clearly associated with mortality ${ }^{1,4,5}$. However, driving pressure, expressed as tidal volume adjusted for compliance, is strongly associated with mortality in ARDS $^{6,7}$. 
COVID-19-induced pneumonia leads to an ARDS-like state with variable infiltrates and profound hypoxia which is typically treated with ARDS mechanical ventilation strategies (e.g., low tidal volumes, high FiO2, high positive end expiratory pressure [PEEP], prone positioning) ${ }^{3,8}$. This has increased interest in lung compliance with studies attempting to elucidate the pathophysiology and heterogeneity of the respiratory failure caused by COVID-19. Although early studies suggested COVID-19-induced pneumonia had preserved static lung compliance, unlike conventional $\operatorname{ARDS}^{9-11}$, recent reports have challenged this ${ }^{12}$.

Although lung compliance and driving pressure may predict outcomes in non-COVID ARDS, the relationship between these variables and outcomes in COVID-19 ARDS is unknown ${ }^{13}$. Further, the relationship between compliance, driving pressure, and time spent on NIV prior to intubation has not been studied. Similarly, the evolution of static lung compliance and driving pressure while on invasive mechanical ventilation has not been well described in COVID-19 ARDS.

The lack of large clinical trials and/or evidence-based guidelines has led to an empiric approach to most aspects of COVID-19 ARDS management ${ }^{14}$. This includes observational and retrospective data which have used NIV as a first line therapy ${ }^{15-18}$. The use of Noninvasive respiratory support in the COVID-19 era has also been driven by practical limitations including personnel shortages, lack of equipment, availability of intensive care unit [ICU] beds, etc. Moreover, the cohort of so-called 'happy hypoxemic' patients - those with high oxygen needs but no signs of respiratory distress - may have lured us into a false sense of security regarding delayed intubation ${ }^{19,20}$. There is also observational evidence supporting the cautious use of NIV to delay mechanical ventilatory support in non-COVID-19 ARDS 21-25.

Given these fragmented and sometimes conflicting data, this study was conducted to assess the effect of the duration of noninvasive respiratory support prior to intubation (i.e., NIV, HFNC, or a combination of NIV and HFNC) on static compliance and driving pressure and retrospectively describe its trajectory over time for COVID-19 and non-COVID-19 ARDS while on mechanical ventilation. Finally, we assessed differences in mortality and length of stay relative to ARDS etiology, duration of noninvasive respiratory support, baseline compliance, and baseline driving pressure.

\section{Methods}

\section{Study Design and Patient Cohort}

We retrospectively identified all mechanically ventilated patients within eCareManager v. 4.1.1 (Philips Healthcare) from January 2019 through September 2020 who had an ARDS diagnosis within any of five Catholic Health Initiative-affiliated hospitals (four in Nebraska; one in lowa). One Nebraska hospital is a university-affiliated academic medical center, one Nebraska hospital is a rural magnet hospital, whereas the remaining three hospitals are suburban community facilities. Prior to data abstraction, we limited the 2019 data to January through September to have identical study periods in 2019 and 2020. All included patients were age of majority for their respective state (19 years of age in Nebraska; 18 years of age in lowa) and only the first hospitalization per patient was included; patients who were transferred between 
facilities were included as one continuous hospitalization. All required ventilator data was abstracted manually. Included patients were divided into three groups: (1) all COVID-19 positive patients, (2) COVID19 negative but culture positive patients, and (3) COVID-19 negative and culture negative patients (see supplemental materials). Patients were considered culture positive if their blood and/or sputum bacterial cultures and/or their viral respiratory panel was positive. The study was approved as exempt research by the Institutional Review Board at Creighton University (InfoEd record number: 2001570).

\section{Outcomes}

The primary outcomes were static lung compliance and driving pressure. Static compliance was calculated as tidal volume [TV] divided by the difference between airway plateau pressure [PPL] and PEEP, whereas driving pressure was calculated as PPL minus PEEP. These values were derived from mandatory ventilator checks by respiratory therapists at 0700,1500 , and 2300 hours over the entirety of the intubation window. Secondary outcomes included in-hospital morality and hospital length of stay.

\section{Statistical Analysis}

Descriptive statistics were stratified by COVID-19 and culture positive/negative status. Depending on data distribution, continuous variables are presented as mean and standard deviation or median and interquartile range, with group differences evaluated using one-way analysis of variance or Kruskal-Wallis test, respectively. Categorical variables are presented as percent and compared using the chi-square test or Fisher's exact test. Static compliance and driving pressure were modeled using linear mixed effects models to account for the correlation inherent to the repeated measurements from the same patient. Between-patient differences in change across ventilator checks was estimated via random slope variance; bias in standard errors resulting from remaining residual heteroscedasticity was accounted for using a likelihood-based empirical estimator. Adjusted models controlled for APACHE IV score on admission to the ICU, body mass index [BMI], and biological sex. The functional form of fixed effects across ventilator checks and days of noninvasive respiratory support prior to intubation were evaluated using restricted cubic splines with pre-specified knot point at the 5th, 35th, 65th, and 95th percentiles. The need for random effects and nonlinear fixed effects were determined using the likelihood ratio test. Between-group differences in change in compliance across ventilator checks was estimated by a fixed group-by-ventilator check cross-level interaction effect. Time-to-in-hospital mortality and risk of inhospital mortality were modeled using Kaplan-Meier curves and Cox proportional hazards model. Proportional hazards were assessed using log-negative-log survival curves and Schoenfeld residuals. To account for censoring due to in-hospital mortality, length of stay was evaluated as probability of discharge using the Kaplan-Meier method and log-rank test. All analyses were conducted using SAS v. 9.4 with $p<.05$ used to indicate statistical significance.

\section{Funding Source}

No funding was used for this study

\section{Results}


A total of 589 patients met inclusion criteria, of whom 55 (9.3\%) were COVID-19 positive, 137 (23.3\%) were COVID-19 negative but culture positive, and 397 (67.4\%) were COVID-19 negative but culture negative. Of the 55 COVID-19 positive patients, 24 (43.6\%) were also culture positive. All outcomes were statistically similar between COVID-19 patients who were culture positive or culture negative; as such, we collapsed COVID-19 positive patients into a single COVID-19 positive group (see supplemental materials for outcomes stratified by culture positive and negative in COVID-19 positive patients). Of the COVID-19 negative but culture positive patients, 11 (8.0\%) had two or more positive cultures.

Demographic and clinical characteristics of each group are provided in Table 1. Compared to COVID-19 negative patients, COVID-19 patients averaged greater APACHE IV scores, BMI, and had a higher rate of steroid use, whereas culture positive patients had greater rate of dialysis compared to COVID-19 or culture negative patients; all other characteristics were statistically similar between groups.

\section{Static Compliance}

COVID-19 patients required invasive ventilation for the longest period during their hospital stay and therefore had the largest number of ventilator checks (Table 2). When modeling change in static compliance across ventilator checks, a statistically significant ventilator check-by-group fixed interaction effect was observed $(p=.011)$ indicating that change in static compliance across ventilator checks differed between groups (Figure 1). Static compliance of COVID-19 patients decreased nonsignificantly by an average of 0.08 -units per ventilator check ( $95 \% \mathrm{Cl}:-0.21$ to $0.05, p=.241)$, whereas statistically significant increases in static compliance per ventilator check were observed in both culture positive patients (slope: $0.12,95 \% \mathrm{Cl}: 0.01-0.24, p=.042$ ) and culture negative patients (slope: $0.18,95 \% \mathrm{Cl}$ : 0.05 $0.32, p=.007)$. The between-group difference in change was statistically significant for COVID-19 patients compared to both culture positive patients (difference $=0.20,95 \% \mathrm{Cl}: 0.03-0.37, p=.022$ ) and culture negative patients $(0.26,95 \% \mathrm{Cl}$ : 0.08-0.44, $p=.005)$, whereas no difference was observed between culture positive and culture negative patients (difference $=0.06,95 \% \mathrm{Cl}:-0.11$ to $0.34, p=.481$ ). These results were maintained after adjusting for APACHE IV score, BMI, and biological sex (Figure 1), with group-specific change in static compliance being constant across APACHE IV scores (ventilator check-bygroup-by-APACHE IV interaction $p=.625$ ), BMI (ventilator check-by-group-by-BMI interaction $p=.562$ ), and biological sex (ventilator check-by-group-by-sex interaction $p=.840$ ).

Use of noninvasive respiratory support was highest in COVID-19 patients compared to both culture positive and culture negative patients (Table 2). Each additional day of pre-intubation noninvasive respiratory support was associated with overall static compliance being lower by an average of 0.60-units (95\% Cl: 0.09 to $1.12, p=.022$ ); this effect did not differ across patient groups (days of noninvasive respiratory support-by-group interaction $p=.357$ ) nor did it differ across ventilator checks (days of noninvasive respiratory support-by-ventilator check interaction $p=.637$ ).

\section{Driving Pressure}


When modeling driving pressure across ventilator checks, a statistically significant ventilator check-bygroup interaction was observed $(p=.014)$ indicating that change in driving pressure differed between groups (Figure 1). Specifically, there was a statistically significant difference in linear change in driving pressure between the COVID-19 and the Culture Negative groups (0.04 vs. -0.03 , respectively; difference $=$ $0.07,95 \% \mathrm{Cl}: 0.02-0.13, p=.011)$ and between the COVID-19 group and the Culture Positive group (0.04 vs. -0.03 , respectively; difference $=0.07,95 \% \mathrm{Cl}: 0.02-0.12, p=.005)$. There was no difference in change between the Culture Negative and Culture Positive groups (difference $=0.00,95 \% \mathrm{Cl}:-0.03$ to $0.04, p=$ .918). A similar set of results were observed after adjusting for APACHE IV score, BMI, and biological sex (Figure 1). Further, each additional day of pre-intubation noninvasive respiratory support was associated with overall driving pressure being higher by an average of 0.17 -units $(95 \% \mathrm{Cl}: 0.05$ to $0.29, p=.006)$; this effect did not differ across patient groups (days of noninvasive respiratory support-by-group interaction $p$ $=.438$ ) nor did it differ across ventilator checks (days of noninvasive respiratory support-by-ventilator check interaction $p=.333$ ).

\section{In-hospital Mortality and Length of Stay}

The overall in-hospital mortality rate was $29.5 \%$ (95\% Cl: $26.0 \%$ to $33.4 \%)$. Statistically higher mortality rates were observed in COVID-19 patients compared to culture positive patients ( $47.3 \%$ vs. $29.2 \%, p=$ .017 ) and culture negative patients ( $47.3 \%$ vs. $27.2 \%, p=.002)$; no difference was observed between culture positive and culture negative patients ( $p=.653$; see supplemental materials). On average, time-todeath differed between groups (log-rank $p=.029$ ), with statistically significant differences between COVID-19 patients and culture positive patients (median: 24 days vs. 44 days, $p=.049$ ) and between culture positive patients and culture negative patients (median: 44 days vs. 29 days, $p=.009$ ); no difference in time-to-death was observed between COVID-19 patients and culture negative patients $(p=$ .149; see supplemental materials). Divergence in survival probability occurred at hospital day 20 , particularly in COVID-19 patients; therefore, we modeled risk of death using a heaviside function at day 20. As shown in Table 3, prior to hospital day 20, culture positive patients had $44 \%$ lower risk of death compared to culture negative patients ( $95 \% \mathrm{Cl}: 16 \%$ to $63 \%, p=.005)$, with no other differences between groups; beginning with hospital day 20, COVID-19 patients averaged 3.2-times higher risk of death compared to culture negative patients (95\% Cl: 1.2-8.8, $p=.025)$ and 2.5-times higher risk of death compared to culture positive patients (95\% Cl: $1.0-6.1 ; p=.049)$. Finally, hospital length of stay was statistically shorter for culture negative patients compared to both COVID-19 patients (median: 9 days vs. 26 days, $p<.001$ ) and culture positive patients (median: 9 days vs. 19 days, $p<.001$ ); no difference in length of stay was observed between COVID-19 patients and culture positive patients $(p=.664$; see supplemental materials).

\section{Discussion}

In this retrospective analysis of mechanically ventilated patients with ARDS, we found declining static pulmonary compliance and increasing driving pressures in patients with COVID-19 whereas non-COVID19 patients demonstrated improving compliance and decreasing driving pressure. Consistent with other 
investigations, COVID-19 patients had higher mortality and longer length of stay ${ }^{26}$. Lower static lung compliance and higher driving pressure at baseline were associated with increased mortality regardless of COVID status and/or positive culture status. Longer duration of non-invasive respiratory support prior to intubation was associated with lower static lung compliance and higher driving pressures over the first 24 hours of mechanical ventilation regardless of etiology (COVID-19 vs. non-COVID-19) or positive culture status. However, days of pre-mechanical ventilation noninvasive respiratory support was not associated with mortality, nor did it moderate any association between patient group and mortality or compliance/driving pressure and mortality.

The relationship between compliance and driving pressure in this study is intuitive (reduced compliance requires increased driving pressure to provide a given TV) and is consistent with prior observations ${ }^{6}$. Similarly, the baseline compliance values in our study were consistent with those seen by others for COVID-19 ARDS and for non-COVID-19 ARDS 27,5 . However, this study was unique in demonstrating that while compliance gradually improved in non-COVID-19 ARDS patients, compliance steadily worsened in COVID-19 ARDS patients.

Our data also affirm that compliance and driving pressure are associated with mortality in COVID-19 ARDS and in non-COVID-19 ARDS ${ }^{28}$. This is physiologically plausible as higher driving pressures result in more cyclical alveolar stretch, increased transpulmonary pressures, and higher circulating cytokine burden - factors associated with increased mortality in ARDS ${ }^{10}$. However, prospective studies are needed to delineate how static compliance and/or driving pressure might be used proactively to guide adjustments in TV and/or PEEP to improve outcomes ${ }^{7,29}$.

Previous studies have questioned the utility of aggressive noninvasive respiratory support in ARDS 30,31 . These authors cite concerns that NIV and HFNC might improve oxygenation parameters and reduce work of breathing - thereby masking ongoing deterioration in pulmonary physiology - resulting in potentiation of lung injury and/or delayed intubation ${ }^{32}$. Our data similarly calls into question the strategy of using noninvasive respiratory support in ARDS patients. Patients who received noninvasive supportive measures subsequently had lower compliance and higher driving pressures when mechanical ventilation was initiated - as well as increased length of stay. Our results are consistent with prior reports of worse outcomes among patients with severe ARDS treated with noninvasive respiratory support compared to mechanical ventilation ${ }^{33-35}$.

In addition to the limitations inherent to our study's retrospective and observational design, we were unable to delineate compliance into pulmonary and chest wall components. However, when we adjusted our findings for obesity, we found no difference. We were also unable to precisely quantify the duration of time patients spent on noninvasive ventilation (Continuous positive airway pressure, Bilevel positive airway pressure) or HFNC as documentation in the electronic medical record was fragmented across health care systems. Although there was inherent between-provider practice variability, all patients were managed by a single group of academic intensivists with similar practice standards and adherence to 
evidence-based medicine. However, variations in nursing and patient and family preference and changes in code status could have altered the time course of care.

\section{Conclusions}

In COVID-19 and non-COVID-19 ARDS, static compliance and driving pressure are associated with patient outcome. As such, understanding these factors along with their trajectory across the disease course is crucial. Our study showed that compared to non-COVID-19 ARDS, COVID-19 ARDS patients appear to have a more ominous trajectory, presumably due to progressive lung injury from the underlying pathologic process and mechanical ventilator-induced injury. Furthermore, additional prospective research is needed regarding the role and effect of non-invasive respiratory support in ARDS as our study suggests that though there is no direct association between the use of non-invasive respiratory support and mortality, its use may be associated with worsening compliance once a patient progresses to needing invasive mechanical ventilation.

\section{Abbreviations}

NIV- Noninvasive ventilation

HFNC- High flow nasal cannula

ARDS- acute respiratory distress syndrome

PEEP- Positive end expiratory pressure

ICU- Intensive care unit

TV- Tidal volume

PPL- Plateau pressure

BMI- Body mass index

\section{Declarations}

Ethics approval and consent to participate: This study was approved by the Creighton University Institutional review board

Consent for publication: Not applicable

Availability of data and material: Data is available on request 
Conflicts of interest/Competing interests: All authors have no conflicts of interest to disclose

Funding: No funding source was used

Authors' contributions: All authors contributed to the study conception and design. Material preparation and data collection were performed by Nikhil Jagan, Robert Plambeck, lan Ng, Jasmin Chovatiya, Jeffrey Macaraeg, Karson Kalian, Zachariah Wittenberg, William Pruett, Jonathan Knedler, Lauren Klein, Pooja Kasinath, Emily Dyer and Adam Bergh, and analysis was performed by Ryan Walters. The first draft of the manuscript was written by Nikhil Jagan, Lee Morrow, Ryan Walters, Robert Plambeck and Mark Malesker. All authors commented on previous versions of the manuscript. All authors read and approved the final manuscript.

Acknowledgements: Not applicable

\section{References}

1. -Ashbaugh D, Bigelow DB, Petty T, Levine B. Acute respiratory distress in adults. The Lancet. 1967 Aug 12;290(7511):319-23.

2. -Tobin MJ. Advances in mechanical ventilation. New England Journal of Medicine. 2001 Jun 28;344(26):1986-96.

3. -Acute Respiratory Distress Syndrome Network. Ventilation with lower tidal volumes as compared with traditional tidal volumes for acute lung injury and the acute respiratory distress syndrome. New England Journal of Medicine. 2000 May 4;342(18):1301-8.

4. -Bernard GR, Artigas A, Brigham KL, Carlet J, Falke K, Hudson L, Lamy M, Legall JR, Morris A, Spragg R. The American-European Consensus Conference on ARDS. Definitions, mechanisms, relevant outcomes, and clinical trial coordination. American journal of respiratory and critical care medicine. 1994 Mar;149(3):818-24.

5. -Force AD, Ranieri VM, Rubenfeld GD, Thompson BT, Ferguson N, Caldwell E, Fan E, Camporota L, Slutsky AS. Acute respiratory distress syndrome. Jama. 2012 Jun 20;307(23):2526-33.

6. -Amato MB, Meade MO, Slutsky AS, Brochard L, Costa EL, Schoenfeld DA, Stewart TE, Briel M, Talmor $D$, Mercat A, Richard JC. Driving pressure and survival in the acute respiratory distress syndrome. New England Journal of Medicine. 2015 Feb 19;372(8):747-55.

7. -Aoyama H, Pettenuzzo T, Aoyama K, Pinto R, Englesakis M, Fan E. Association of driving pressure with mortality among ventilated patients with acute respiratory distress syndrome: a systematic review and meta-analysis. Critical care medicine. 2018 Feb 1;46(2):300-6.

8. -Guérin C, Reignier J, Richard JC, Beuret P, Gacouin A, Boulain T, Mercier E, Badet M, Mercat A, Baudin $\mathrm{O}$, Clavel M. Prone positioning in severe acute respiratory distress syndrome. New England Journal of Medicine. 2013 Jun 6;368(23):2159-68.

9. -Gattinoni L, Coppola S, Cressoni M, Busana M, Rossi S, Chiumello D. COVID-19 does not lead to a "typical" acute respiratory distress syndrome. American journal of respiratory and critical care 
medicine. 2020 May 15;201(10):1299-300.

10. -Chiumello D, Busana M, Coppola S, Romitti F, Formenti P, Bonifazi M, Pozzi T, Palumbo MM, Cressoni M, Herrmann P, Meissner K. Physiological and quantitative CT-scan characterization of COVID-19 and typical ARDS: a matched cohort study. Intensive care medicine. 2020 Dec;46(12):2187-96.

11. -Grasselli G, Tonetti T, Protti A, Langer T, Girardis M, Bellani G, Laffey J, Carrafiello G, Carsana L, Rizzuto C, Zanella A. Pathophysiology of COVID-19-associated acute respiratory distress syndrome: a multicentre prospective observational study. The lancet Respiratory medicine. 2020 Dec 1;8(12):1201-8.

12. -Ferrando C, Suarez-Sipmann F, Mellado-Artigas R, Hernández M, Gea A, Arruti E, Aldecoa C, MartínezPallí G, Martínez-González MA, Slutsky AS, Villar J. Clinical features, ventilatory management, and outcome of ARDS caused by COVID-19 are similar to other causes of ARDS. Intensive care medicine. 2020 Dec;46(12):2200-11.

13. -Panwar R, Madotto F, Laffey JG, Van Haren FM. Compliance phenotypes in early acute respiratory distress syndrome before the COVID-19 pandemic. American journal of respiratory and critical care medicine. 2020 Nov 1;202(9):1244-52.

14. - Tsang JL, Binnie A, Fowler RA. Twenty articles that critical care clinicians should read about COVID19. Intensive Care Medicine. 2021 Mar;47(3):337-41.

15. -World Health Organization. Clinical management of severe acute respiratory infection when novel coronavirus (2019-nCoV) infection is suspected: interim guidance. InClinical management of severe acute respiratory infection when novel coronavirus (2019-nCoV) infection is suspected: Interim guidance 2020 (pp. 21-21).

16. -Alhazzani W, Møller MH, Arabi YM, Loeb M, Gong MN, Fan E, Oczkowski S, Levy MM, Derde L, Dzierba A, Du B. Surviving Sepsis Campaign: guidelines on the management of critically ill adults with Coronavirus Disease 2019 (COVID-19). Intensive care medicine. 2020 May;46(5):854-87.

17. -Wang D, Hu B, Hu C, Zhu F, Liu X, Zhang J, Wang B, Xiang H, Cheng Z, Xiong Y, Zhao Y. Clinical characteristics of 138 hospitalized patients with 2019 novel coronavirus-infected pneumonia in Wuhan, China. Jama. 2020 Mar 17;323(11):1061-9.

18. -Yang X, Yu Y, Xu J, Shu H, Liu H, Wu Y, Zhang L, Yu Z, Fang M, Yu T, Wang Y. Clinical course and outcomes of critically ill patients with SARS-CoV-2 pneumonia in Wuhan, China: a single-centered, retrospective, observational study. The Lancet Respiratory Medicine. 2020 May 1;8(5):475-81.

19. -Dhont S, Derom E, Van Braeckel E, Depuydt P, Lambrecht BN. The pathophysiology of ‘happy’hypoxemia in COVID-19. Respiratory research. 2020 Dec;21(1):1-9.

20. -Machado-Curbelo C. Silent or'Happy'Hypoxemia: An Urgent Dilemma for COVID-19 Patient Care. MEDICC review. 2021 Jul 7;22:85-6.

21. -Confalonieri M, Potena A, Carbone G, Porta RD, Tolley EA, Umberto Meduri G. Acute respiratory failure in patients with severe community-acquired pneumonia: a prospective randomized evaluation 
of noninvasive ventilation. American Journal of Respiratory and Critical Care Medicine. 1999 Nov 1;160(5):1585-91.

22. -Ferrer M, Esquinas A, Leon M, Gonzalez G, Alarcon A, Torres A. Noninvasive ventilation in severe hypoxemic respiratory failure: a randomized clinical trial. American journal of respiratory and critical care medicine. 2003 Dec 15;168(12):1438-44.

23. -Thille AW, Contou D, Fragnoli C, Córdoba-Izquierdo A, Boissier F, Brun-Buisson C. Non-invasive ventilation for acute hypoxemic respiratory failure: intubation rate and risk factors. Critical Care. 2013 Dec;17(6):1-8.

24. -Carrillo A, Gonzalez-Diaz G, Ferrer M, Martinez-Quintana ME, Lopez-Martinez A, Llamas N, Alcazar M, Torres A. Non-invasive ventilation in community-acquired pneumonia and severe acute respiratory failure. Intensive care medicine. 2012 Mar;38(3):458-66.

25. -Antonelli M, Conti G, Rocco M, Bufi M. Deblasi RA, Vivino G, Gasparetto A, Meduri GV. A comparison of noninvasive positive-pressure ventilation and conventional mechanical ventilation in patients with acute respiratory failure. N Engl J Med. 1998;339:429-35.

26. -Higgins TL, Stark MM, Henson KN, Freeseman-Freeman L. Coronavirus Disease 2019 ICU Patients Have Higher-Than-Expected Acute Physiology and Chronic Health Evaluation-Adjusted Mortality and Length of Stay Than Viral Pneumonia ICU Patients. Critical care medicine. 2021 Jul 1;49(7):e701-6.

27. -Bassi GL, Suen JY, Dalton HJ, White N, Shrapnel S, Fanning JP, Liquet B, Hinton S, Vuorinen A, Booth G, Millar JE. An appraisal of respiratory system compliance in mechanically ventilated covid-19 patients. Critical Care. 2021 Dec;25(1):1-22.

28. -Zanella A, Florio G, Antonelli M, Bellani G, Berselli A, Bove T, Cabrini L, Carlesso E, Castelli GP, Cecconi M, Citerio $\mathrm{G}$. Time course of risk factors associated with mortality of 1260 critically ill patients with COVID-19 admitted to 24 Italian intensive care units. Intensive care medicine. 2021 Aug 9:1-4.

29. -Yoshida T, Fujino Y, Amato MB, Kavanagh BP. Fifty years of research in ARDS. Spontaneous breathing during mechanical ventilation. Risks, mechanisms, and management. American journal of respiratory and critical care medicine. 2017 Apr 15;195(8):985-92.

30. -Kang BJ, Koh Y, Lim CM, Huh JW, Baek S, Han M, Seo HS, Suh HJ, Seo GJ, Kim EY, Hong SB. Failure of high-flow nasal cannula therapy may delay intubation and increase mortality. Intensive care medicine. 2015 Apr;41(4):623-32.

31. -Frat JP, Thille AW, Mercat A, Girault C, Ragot S, Perbet S, Prat G, Boulain T, Morawiec E, Cottereau A, Devaquet J. High-flow oxygen through nasal cannula in acute hypoxemic respiratory failure. New England Journal of Medicine. 2015 Jun 4;372(23):2185-96.

32. -Brochard L, Slutsky A, Pesenti A. Mechanical ventilation to minimize progression of lung injury in acute respiratory failure. American journal of respiratory and critical care medicine. $2017 \mathrm{Feb}$ 15;195(4):438-42.

33. -AntonelliM CG. A multiple2center survey on the use in clinical practice of noninvasive ventilation as a first2 line intervention for acute respiratory distress syndrome. Crit Care Med. 2007;35:18-25. 
34. -Rana S, Jenad H, Gay PC, Buck CF, Hubmayr RD, Gajic O. Failure of non-invasive ventilation in patients with acute lung injury: observational cohort study. Critical Care. 2006 Jun;10(3):1-5.

35. -Bellani G, Laffey JG, Pham T, Madotto F, Fan E, Brochard L, Esteban A, Gattinoni L, Bumbasirevic V, Piquilloud L, Van Haren F. Noninvasive ventilation of patients with acute respiratory distress syndrome. Insights from the LUNG SAFE study. American journal of respiratory and critical care medicine. 2017 Jan 1;195(1):67-77.

\section{Tables}

Table 1. Demographic and clinical characteristics

\begin{tabular}{|c|c|c|c|c|}
\hline & $\begin{array}{l}\text { COVID-19 } \\
(n=55)\end{array}$ & $\begin{array}{l}\text { Culture Positive } \\
(n=137)\end{array}$ & $\begin{array}{l}\text { Culture Negative } \\
(n=397)\end{array}$ & $p$ \\
\hline Age & $61.4 \pm 13.7$ & $60.4 \pm 15.4$ & $58.7 \pm 18.4$ & 0.392 \\
\hline \multicolumn{5}{|c|}{ Biological Sex } \\
\hline Female & 47.3 & 42.3 & 41.7 & \multirow[t]{2}{*}{0.733} \\
\hline Male & 52.7 & 57.7 & 58.3 & \\
\hline Height & $166.5 \pm 9.3$ & $170.6 \pm 11.4$ & $171.1 \pm 11.5$ & 0.223 \\
\hline Weight & $92.1 \pm 24.8$ & $88.7 \pm 25.4$ & $89.4 \pm 26.0$ & 0.446 \\
\hline BMI & $33.3 \pm 8.9$ & $30.5 \pm 8.4$ & $29.8 \pm 8.3$ & 0.021 \\
\hline APACHE & 102 [75-117] & 93 [73-118] & 85 [65-107] & 0.017 \\
\hline \multicolumn{5}{|c|}{ Comorbidities } \\
\hline Asthma & 5.9 & 8.3 & 9.9 & 0.598 \\
\hline CHF & 7.8 & 21.8 & 17.7 & 0.085 \\
\hline CKD & 15.7 & 24.1 & 22.9 & 0.454 \\
\hline COPD & 11.8 & 22.6 & 20.4 & 0.256 \\
\hline DM & 45.1 & 31.6 & 29.6 & 0.082 \\
\hline Dialysis & 5.9 & 12.8 & 5.6 & 0.022 \\
\hline HTN & 62.8 & 63.4 & 56.3 & 0.288 \\
\hline OSA & 23.5 & 18.1 & 14.2 & 0.173 \\
\hline Steroids & 25.5 & 5.2 & 0.5 & $<.001$ \\
\hline
\end{tabular}


Note. Data presented as mean \pm SD, median [IQR], or percent. Note that the COVID-19 group represents all COVID-19 positive patients, the Culture Positive group represents COVID-19 negative patients with positive blood, sputum, and/or other viral culture, and the Culture Negative group represents COVID-19 negative patients with negative blood, sputum, and other viral culture.

Table 2. Invasive and noninvasive respiratory support

$$
\begin{array}{lll}
\text { COVID-19 } & \text { Culture Positive } & \text { Culture Negative } \\
(n=55) & (n=137) & (n=397)
\end{array}
$$

\begin{tabular}{|c|c|c|c|c|}
\hline Total Ventilator Checks & 26 [16-52] & 16 [7-33] & $7[4-13]$ & $<.001$ \\
\hline Total Days & 9 [6-18] & $6[3-11]$ & $3[2-5]$ & $<.001$ \\
\hline \multicolumn{5}{|c|}{ Noninvasive Respiratory Support } \\
\hline No & 60.0 & 84.7 & 88.9 & \multirow[t]{2}{*}{$<.001$} \\
\hline Yes & 40.0 & 15.3 & 11.1 & \\
\hline NIPPV & 27.3 & 55.0 & 60.5 & \multirow[t]{3}{*}{0.031} \\
\hline HFNC & 27.3 & 35.0 & 23.3 & \\
\hline NIPPV + HFNC & 45.5 & 10.0 & 16.3 & \\
\hline Total Days & 2 [1-4] & 1 [1-2] & 1 [1-2] & 0.040 \\
\hline
\end{tabular}

Invasive Ventilation

Note. Data presented as median [interquartile range] or percent. Note that the COVID-19 group represents all COVID-19 positive patients, the Culture Positive group represents COVID-19 negative patients with positive blood, sputum, and/or other viral culture, and the Culture Negative group represents COVID-19 negative patients with negative blood, sputum, and other viral culture.

Table 3. Hazard ratios (HR) for risk of in-hospital death comparing groups $<20$ days and $\geq 20$ days

\begin{tabular}{llllll} 
& LOS $<20$ Days & & LOS $\geq 20$ Days & & Difference \\
\hline & HR $(95 \% \mathrm{Cl})$ & $p$ & HR $(95 \% \mathrm{Cl})$ & $p$ & $p$ \\
\hline COVID-19 vs. Negative & $0.65(0.38-1.10)$ & 0.110 & $3.20(1.16-8.83)$ & 0.025 & 0.006 \\
\hline COVID-19 vs. Positive & $1.16(0.64-2.12)$ & 0.626 & $2.47(1.00-6.10)$ & 0.049 & 0.172 \\
\hline Positive vs. Negative & $0.56(0.37-0.84)$ & 0.005 & $1.29(0.46-3.64)$ & 0.626 & 0.138
\end{tabular}

$\mathrm{HR}=$ hazard ratio 
Note. The group after the "vs." is the reference. So, a HR > 1 implies greater risk of death compared to the reference. For example, in those with LOS $\geq 20$ days, COVID-19 patients averaged 3.2-times greater risk of death relative to culture negative patients. The "Difference" column compares the risk of death $<20$ compared to $\geq 20$ days. Note that the COVID-19 group represents all COVID-19 positive patients, the Positive group represents COVID-19 negative patients with positive blood, sputum, and/or other viral culture, and the Negative group represents COVID-19 negative patients with negative blood, sputum, and other viral culture.

\section{Figures}
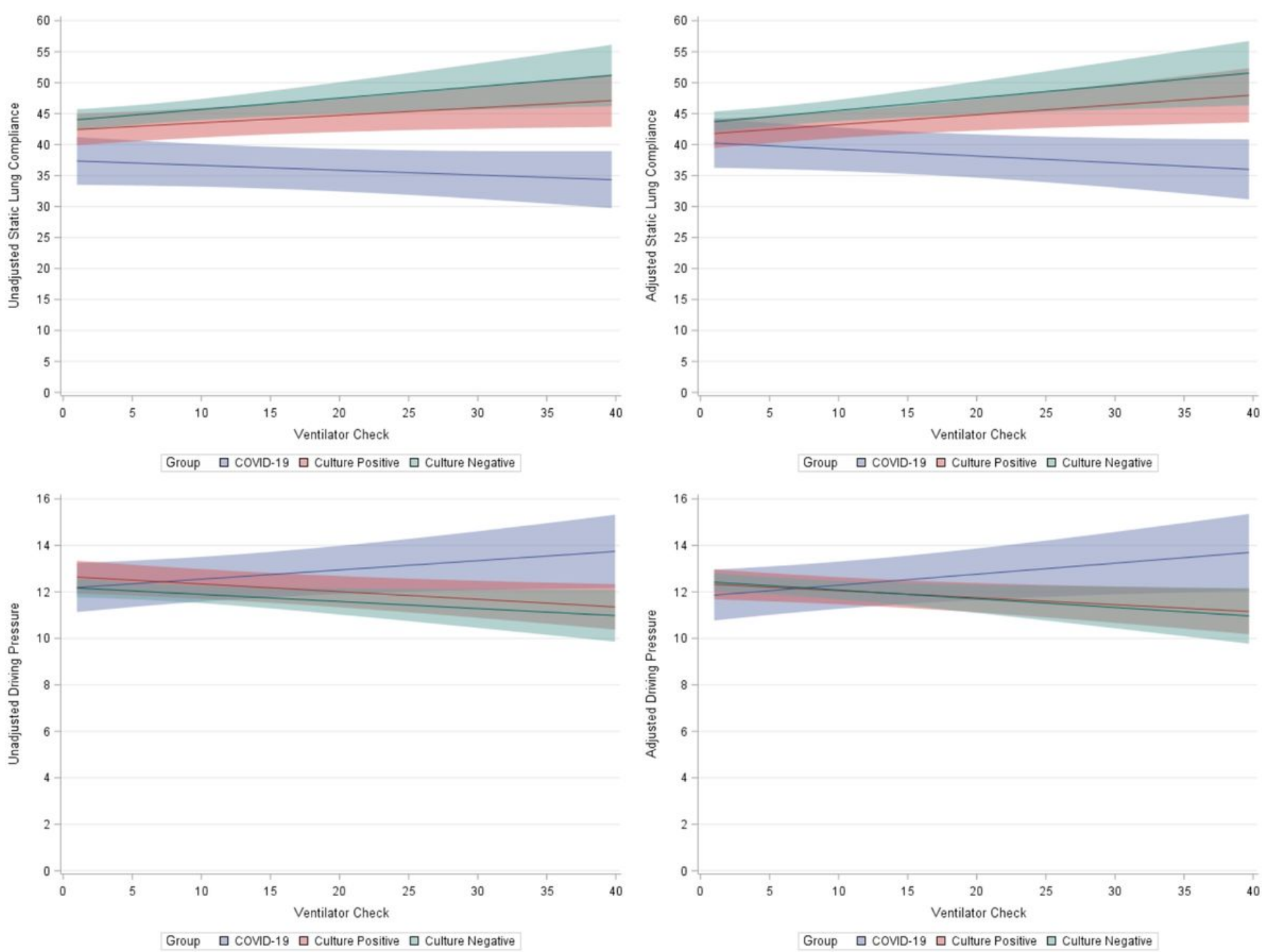

\section{Figure 1}

Unadjusted (left column) and adjusted (right column) estimated static compliance (top row) and driving pressure (bottom row) across ventilator checks; estimates were adjusted for BMI and biological sex. Although the statistical models were estimated using static compliance or driving pressure at all 
available ventilator checks, in these figures, the number of ventilator checks was truncated at 40 given that $95 \%$ of culture negative patients had $\leq 42$ ventilator checks. Shaded areas represent $95 \%$ confidence intervals. Note that the COVID-19 group represents all COVID-19 positive patients, the Culture Positive group represents COVID-19 negative patients with positive blood, sputum, and/or other viral culture, and the Culture Negative group represents COVID-19 negative patients with negative blood, sputum, and other viral culture.

\section{Supplementary Files}

This is a list of supplementary files associated with this preprint. Click to download.

- SupplementTimedStudy.docx 